\title{
Vibrational response of adaptive composites
}

\author{
M. Parlinska, J.A. Balta', V. Michaud, J.-E. Bidaux², J.A. Månson ${ }^{1}$ \\ and R. Gotthardt
}

Institut de Génie Atomique, Département de Physique, EPFL, 1015 Lausanne, Switzerland

${ }^{1}$ Laboratoire de Technologie des Composites et Polymères, Département des Matériaux, EPFL, 1015 Lausanne, Switzerland

${ }^{2}$ Groupe Matériaux et Conception, route du Rawyl 47, École d'Ingénieurs du Valais, 1950 Sion, Switzerland

\begin{abstract}
Composite laminates containing pre-deformed NiTiCu wires embedded in an epoxy matrix reinforced with Kevlar fibres were manufactured and tested. These materials change their properties, for example vibration resonance frequency or modulus in response to a temperature variation. When heated by direct electrical current above the transformation temperature, the pre-deformed shape memory alloy (SMA) wires try to recover their shape and since they are restrained by a stiff matrix and clamping, a stress is created. As a result, a change in the resonance frequency of the composite occurs. The magnitude of the recovery stress and corresponding resonance frequency shift was found to increase with the SMA wire volume fraction and to decrease with the thickness of the host composite layers between the wires and the constraining grips.
\end{abstract}

\section{INTRODUCTION}

In the past years, shape memory alloys (SMA) in the form of wires, short fibres or thin-films have found an increasing interest as thermally activated actuators embedded in polymer matrix composites (SMAcomposites) [1]. Such SMA-composites offer the potential to actively control elastic modulus, internal stress-state and natural vibration frequencies in case of hard matrices and to induce a shape change in case of soft embedment, once the composite is produced [2,3].

Numerous models have been developed describing the transformation behaviour of embedded SMA wires, the transformation strain and the thermal expansion coefficient [4,5]. However, these models have not often been confronted to experimental data. Nevertheless, shifts to higher resonance frequencies have been observed experimentally and stress distribution has been studied using finite elements by Bidaux $e t$ al. [6,7]. They investigated a simple model system consisting of SMA wires embedded in an epoxy matrix without any other reinforcement.

In the framework of a European research project, adaptive composites based on Kevlar-epoxy host composite materials with embedded NiTi-based SMA wires are currently under investigation. NiTiCu wires were found to exhibit a small hysteresis, comparable to that of NiTi in R-phase and yet to be less pre-strain sensitive [8,9]. This paper presents experimental results on the influence of the geometry of composite laminates and the volume fraction of $\mathrm{Ni} T \mathrm{TiCu}$ wires on the magnitude of the resonance frequency shift and of the recovery force.

\section{MATERIALS AND EXPERIMENTAL TECHNIQUES}

The NiTiCu wires, $150 \mu \mathrm{m}$ in diameter, are supplied by Raychem. The alloy contains about 12 wt. pct copper substituting the $\mathrm{Ni}$. The host composite was delivered as prepregs of unidirectional aligned Kevlarß 29 aramid fibers and LTM217 epoxy resin, manufactured by Advanced Composite Group in UK. Composite manufacturing was as follows: the wires were placed in a specially designed frame, heated to $100^{\circ} \mathrm{C}$ to release potential deformation of the wire during handling, and pre-strained by the desired amount. Plies of prepregs were placed on each side of the wires, as shown schematically in Fig. 1 . The wire pre-strain was maintained by the frame during curing in an autoclave, restraining the wires from recovering their shape. The resin was cured for 12 hours at $70^{\circ} \mathrm{C}$ under vacuum with a heat up rate of 


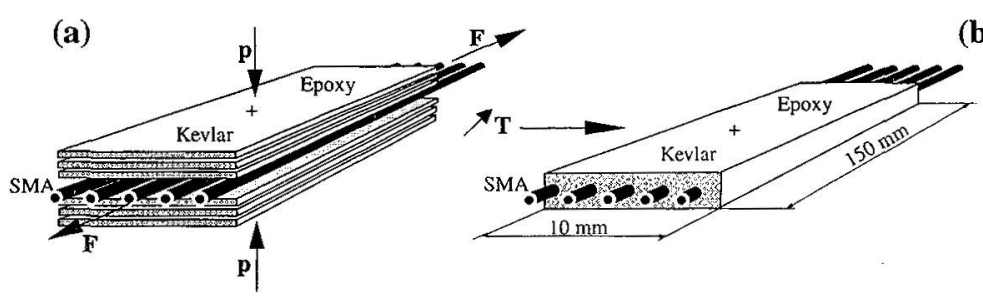

Fig. 1. Schematic drawing of the fabrication process of the SMA composite with SMA wires embedded in epoxy matrix with Kevlar fibres. (b)

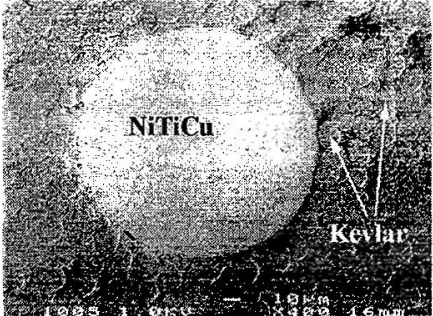

Fig. 2. SEM photo of a transverse cut of the composite sample.

$4^{\circ} \mathrm{C} / \mathrm{min}$. The post-curing was 1 hour at $140^{\circ} \mathrm{C}$ with a heating ramp of $20^{\circ} \mathrm{C} /$ hour resulting in a glass transition temperature of the matrix of $\mathrm{T}_{\mathrm{g}} \cong 180^{\circ} \mathrm{C}$. Various samples with one layer of wires and with different spacing between the wires were produced: 4,8 or 16 and 5,10 or 20 wires per $\mathrm{cm}$ of composite, corresponding to a distance of 2,1 and $0.5 \mathrm{~mm}$ between the wires. Samples with two layers of wires separated by two prepreg layers were also produced. A summary of the produced samples is given in Table 1. A scanning electron microscope photograph of a transverse cut through the composite is presented in Fig. 2, showing a homogenous distribution of the Kevlar fibres obtained by this process.

The vibration response of the composite samples activated by electrical current heating was measured using the set-up developed by Bidaux et al. [2]. It is composed of a sample holder, a shaker, a Laser Doppler detector and a computer to record and treat the measured signal. The composites are mounted on a U-shaped sample holder, (Fig. 2), which allows the measurement of the force exerted by the composite

Tab. 1: Inventory of samples used in experiments.

\begin{tabular}{|c|c|c|c|c|c|c|c|c|c|c|c|c|c|c|c|c|c|c|}
\hline Sample & $1 \mathrm{~A}$ & $1 \mathrm{~B}$ & $1 \mathrm{C}$ & $2 \mathrm{~A}$ & $2 B$ & $2 \mathrm{C}$ & $3 \mathrm{~A}$ & $3 \mathrm{~B}$ & $3 \bar{C}$ & $4 \mathrm{~A}$ & $4 B$ & $4 \mathrm{C}$ & $5 \mathrm{~A}$ & $5 \mathrm{~B}$ & $5 \mathrm{C}$ & $6 \mathrm{~A}$ & $6 \mathrm{~B}$ & $6 C$ \\
\hline $\mathrm{Nb}$. of prepregs & \multicolumn{3}{|c|}{$1 / 1(\sim 0.5 \mathrm{~mm})$} & \multicolumn{3}{|c|}{$2 / 2(\sim 0.5 \mathrm{~mm})$} & \multicolumn{3}{|c|}{$3 / 3(\sim 0.7 \mathrm{~mm})$} & \multicolumn{3}{|c|}{$4 / 4(\sim 0.9 \mathrm{~mm})$} & \multicolumn{3}{|c|}{$2 / 2 / 2(\sim 0.7 \mathrm{~mm})$} & \multicolumn{3}{|c|}{$3 / 3(\sim 0.7 \mathrm{~mm})$} \\
\hline $\mathrm{Nb}$ of wires & 4 & 8 & 16 & 4 & 8 & 16 & 4 & 8 & 16 & 4 & 8 & 16 & $4+4$ & $8+8$ & $8+8$ & 5 & 10 & 20 \\
\hline \%of wire prestrain & \multicolumn{3}{|c|}{5.5} & \multicolumn{3}{|c|}{5.5} & \multicolumn{3}{|c|}{5.5} & \multicolumn{3}{|c|}{5.5} & \multicolumn{3}{|c|}{5.5} & \multicolumn{3}{|c|}{3.5} \\
\hline wire vol.frac.[\%] & 3 & 5.8 & 11 & 0.9 & 2.2 & 4.4 & 1 & 1.8 & 3.5 & 0.5 & 1.1 & 2.3 & 2 & 3.15 & 3.3 & 1 & 2 & 4 \\
\hline
\end{tabular}

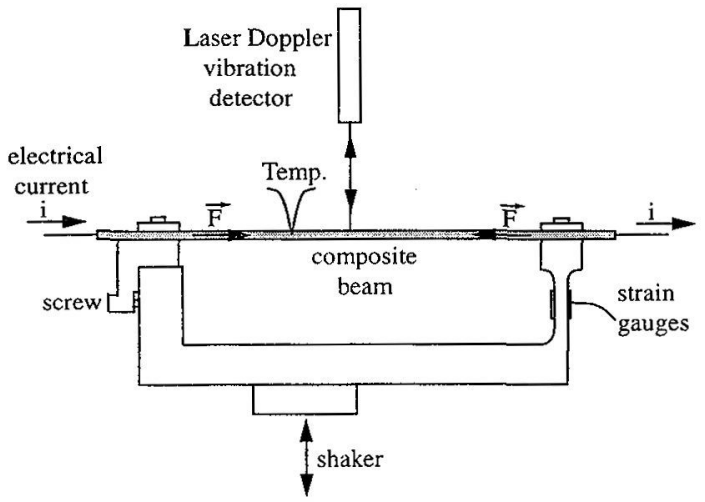

Fig. 3. Schematic drawing of the U-shaped sample holder allowing simultaneous measurements of the strain and frequency response of the composite samples. 
on the grips and the vibration frequency of the beam as a function of temperature. The sample holder is fixed on a shaker, which produces vibrations perpendicular to the plane of the composite. The entire sample can additionally be pre-deformed with a screw placed at one end of the sample holder.

The SMA wires were heated incrementally by direct electrical current and cooled down by convection at the matrix surface after switching off the current. After each intensity increment, the temperature was allowed to stabilise for at least 30 seconds before each force measurement. For frequency measurements the stabilisation time was about 3 minutes because the current increments were larger. The SMA wires were connected in series to obtain a constant current flow in all the fibres and the temperature of the composite was measured at its surface with a $50 \mu \mathrm{m}$ diameter thermocouple. A heat conductive paste ensured a good contact between the thermocouple and the composite surface.

\section{RESULTS AND DISCUSSION}

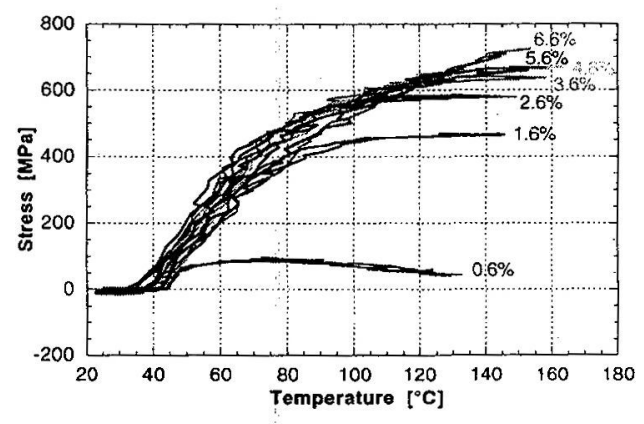

Fig. 4. Recovery stress generated by a neat $\mathrm{NiTiCu}$ wire from $0.6 \%$ to $6.6 \%$ pre-strain.

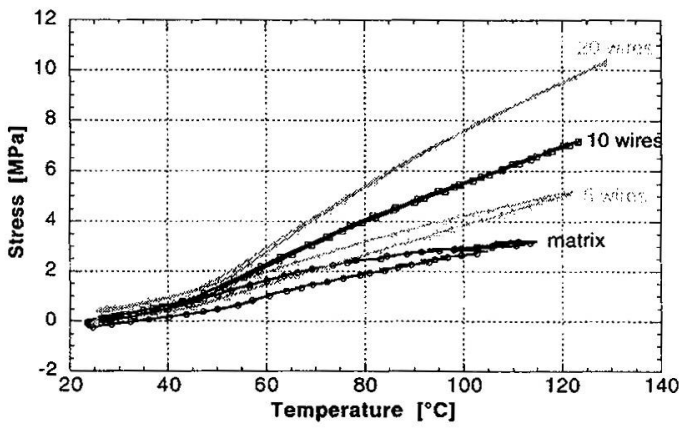

Fig. 5. Dependence of stress on temperature for composite samples: $6 \mathrm{~A}, 6 \mathrm{~B}, 6 \mathrm{C}$ and host composite.

The as-received wires showed an initial strain of $2.5 \%$ probably due to the fabrication process. In addition, a small two-way shape memory effect (TWSME) resulting in a residual strain of $0.6 \%$ was observed [9]. To eliminate the initial strain, the single wires were first heated to $120^{\circ} \mathrm{C}$ and cooled without constraint, then clamped and pre-strained before testing. Figure 4 shows a typical temperature dependence of the recovery stress developed by the wires. The indicated percentage thus corresponds to the amount of imposed pre-strain, plus the residual pre-strain. The most important variation of the force occurred between approximately $40^{\circ} \mathrm{C}$ and $75^{\circ} \mathrm{C}$. The slope of the stress-temperature curve is about $5.6 \mathrm{MPa} /{ }^{\circ} \mathrm{C}$. For the wire without any pre-strain $(0.6 \%)$ a stress of $100 \mathrm{MPa}$ due to the TWSME $(0.6 \%)$ is measured (Figure 4). The small decrease of the stress above the maximum is probably due to thermal expansion. Note that beyond about $3 \%$ pre-strain, the stress response reaches saturation, to a value of about $680 \mathrm{MPa}$.

During composite manufacturing, the wires were not heated before being clamped in the frame, hence any imposed pre-strain was in addition to the $2.5 \%$ initial pre-strain in the as-received wires. The pre-strain values given in Table I take this initial pre-strain into account.

Figure 5 compares the recovery stress generated by composite samples $6 \mathrm{~A}, 6 \mathrm{~B}$ and $6 \mathrm{C}$ containing different number of NiTiCu wires and by the host composite alone. The stress increases with temperature and with the number of embedded wires. This stress change is related to the direct $M \rightarrow A$ phase transformation of the wires. Also, the composite samples exhibit a small hysteresis, which makes them suitable candidates for industrial applications. The host composite alone also shows an increase of stress with temperature, which is due to the negative coefficient of thermal expansion (CTE) of the Kevlar fibres, leading to an overall negative CTE for the host composite. 


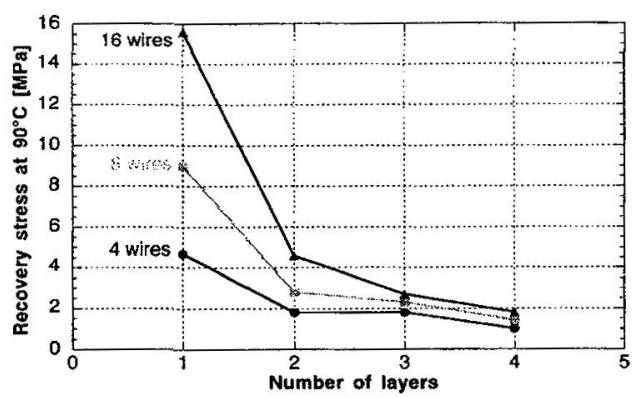

Fig. 6 Dependence of recovery stress measured at $90^{\circ} \mathrm{C}$ on number of pre-preg layers for composite samples 1 Ato $4 \mathrm{C}$.

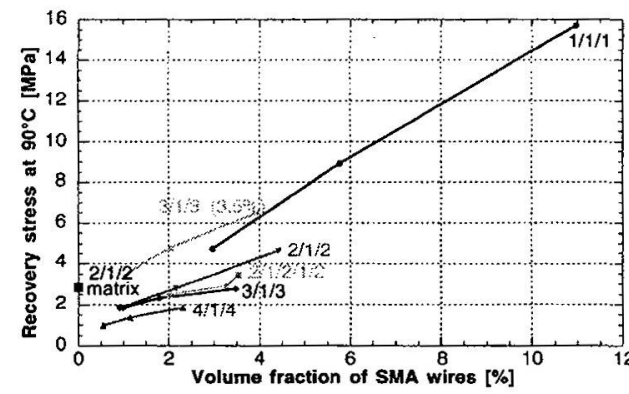

Fig. 7 Dependence of recovery stress measured at $90^{\circ} \mathrm{C}$ on volume fraction of SMA wires for all composite samples and the host composite (matrix).

Recovery stress measured at $90^{\circ} \mathrm{C}$ as a function of number of pre-preg layers is plotted in Figure 6 . As previously described the recovery stress increases with the number of wires. However, the magnitude of the effect strongly decreases with the number of pre-preg layers. A possible explanation is that when the thickness of the host composite increases, the contribution of the interfacial stress between the wire and the host composite is less effective at the location of the measuring points. The same results are presented in Figure 7, in terms of wire volume fraction. The recovery stress for the two wire layer composites ( $5 \mathrm{~A}$ to $\mathrm{C}$ ), for the composite with $3.5 \%$ pre-strain $(6 \mathrm{~A}$ to $\mathrm{C}$ ) and for the host composite alone are plotted alongside. A general trend of increasing recovery stress with volume fraction is clearly observed. In particular, the samples with $3.5 \%$ pre-strain exhibit recovery stresses which are in the same range as those with 5.5\%. This is in agreement with the single wire behaviour (Fig.4), where a saturation of the recovery force beyond $3 \%$ pre-strain was noticed. The behaviour of samples manufactured with two layers of wires is also in the same range as the others. This could be explained based on the results shown in Figure 6: in both cases, two layers of pre-preg between the grips and the wire layers seem to appear as the recovery stress controlling parameter, compared to the effect of the number of wires. Note that the host composite alone exhibits a relatively high recovery stress due to its negative CTE. More experiments are under progress to confirm this result.

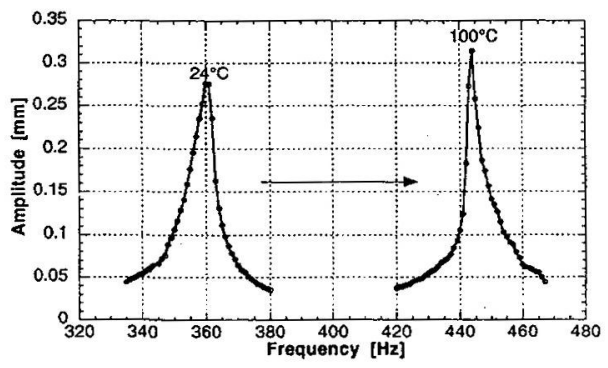

Fig. 8. Resonance frequency shift after activation of composite sample $6 \mathrm{~B}$, measured before the experiment and during cooling, respectively.

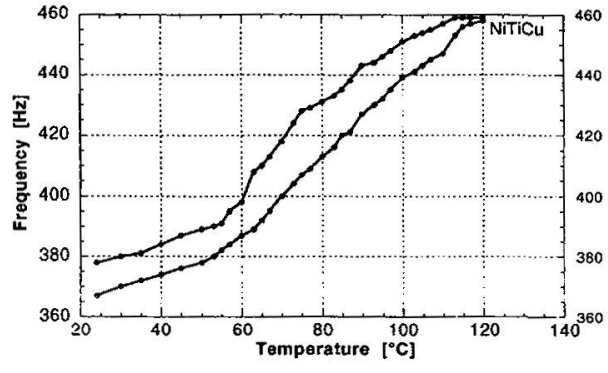

Fig. 9. Evolution of frequency as a function of temperature for composite sample 6B. 


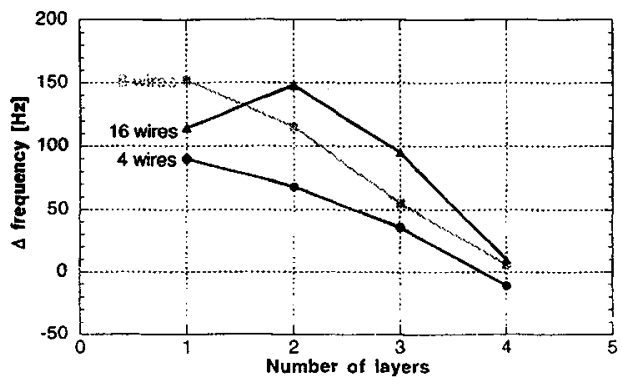

Fig. 10 Resonance frequency shift $v s$ number of prepreg layers for composite samples $1 \mathrm{~A}$ to $4 \mathrm{C}$.

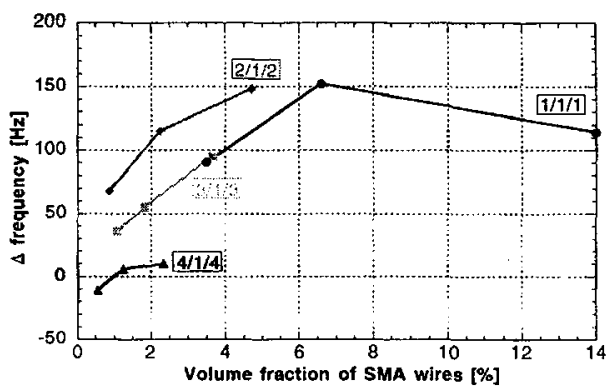

Fig. 11 Resonance frequency shift as a function of SMA wire volume fraction for composite samples 1 Ato $4 \mathrm{C}$,.

For both figures: the resonance frequency shift is measured between $24^{\circ} \mathrm{C}$ and $100^{\circ} \mathrm{C}$.

Figure 8 shows typical resonance peaks of the SMA-composites measured at room temperature before starting the experiment and at $100^{\circ} \mathrm{C}$ during cooling for sample $6 \mathrm{~B}$. A shift of the resonance frequency peak of $85 \mathrm{~Hz}$ is observed. Figure 9 illustrates the evolution of frequency as a function of temperature for the same sample. This frequency-temperature dependence curve roughly follows the curve of recovery force generated by the embedded wires, Figure 4, confirming the relationship previously found for epoxySMA composites [2]. Additional experiments are in progress in order to complete and confirm these results.

Figures 10 and 11 illustrate the shift in the resonance frequency for samples $1 \mathrm{~A}$ to $4 \mathrm{C}$ between $24^{\circ} \mathrm{C}$ and $100^{\circ} \mathrm{C}$ as a function of the number of prepreg layers on each side of the wires and SMA wire volume fraction, respectively. The resuits obtained by measuring the frequency shift as a function of prepreg layers or volume fraction of the SMA wires are in general agreement with the corresponding recovery stress curve. For thin composite samples having only one prepreg layer on each side of the SMA wires, measurements of vibration frequency become difficult due to the buckling of the composite sample after activation. This may explain the decrease of the frequency shift for the sample with one layer of pre-preg on each side of sample $1 \mathrm{C}$ (Fig. 10 and 11) even though the recovery stress increases nearly linearly with the number of wires.

\section{CONCLUSIONS}

Hybrid SMA composites based on Kevlar-epoxy host composite matrix and thin embedded NiTiCu wires were investigated. Composite samples with different amount of wires, different geometrical arrangements and various levels of wires pre-strain were successfully produced. Upon activation, the composites showed a recovery stress and corresponding shift of the resonance frequency to higher values. The wires were found to be suitable for application in adaptive vibration control, as the hysteresis was small and the magnitude of the activation effect reasonably high. It was shown that it is necessary to characterise the as-received wires, in particular to measure their initial strain and possible two-way shape memory behaviour. The as-received NiTiCu wires exhibited an initial pre-strain of $2.5 \%$.

The composite behaviour roughly followed the behaviour of the neat wires, although with a reduced activation magnitude. Activation was found to be an increasing function of the wire volume fraction. However, the geometry of the samples also play an important role. The thinner the layer between the SMA wires and the grips, the higher the magnitude of the observed effect. 


\section{ACKNOWLEDGEMENTS}

The authors are grateful to Ms J.Hudd from British Aerospace Research Centre Sowerby for sample processing and Ms Hélène Clech from IUP matériaux, UFR Sciences, Poitiers for her contribution to the results presented in this paper. This research was made in the frame of the ADAPT Brite/EuRam Project, which is funded by the European Commission in the Industrial and Materials Technologies research and technological program.

\section{REFERENCES}

[1] Z. G. Wei, R. Sandstorm, S. Miyazaki "Shape memory materials and hybrid composites for smart composites" part I and II, Journal of Materials Science, vol. $\underline{33}, \mathrm{n}^{\circ} 15, \mathrm{pp} .3763-83,1998$

[2] J.-E. Bidaux, J.-A.E. Manson, R. Gotthardt "Active stiffening of composite materials by embedded shape memory alloy fibres", Mat. Res. Soc. Symp. Proc. Vol.459, pp. 107-117, 1997

[3] K. Escher, E. Hornbogen, "Aspects of two-way shape memory effect in NiTi-Silicon composite materials" J. de Physique, 1, C4-pp. 427-432, 1991

[4] R. Stalmans, L. Delaey and J. Van Humbeeck, "Modeling of adaptive composite materials with embedded shape memory alloy wires", Mat. Res. Soc. Symp. Proc. Vol.459, pp. 119-130, 1997

[5] J. Wang, Y. P. Shen "Micromechanics of composites reinforced in the aligned SMA short fibres uniform thermal fields", Smart Mat. and Struct., vol. 9 , ${ }^{\circ} 1$, pp.69-77, 2000

[6] J.-E. Bidaux, J.-A. Manson, R. Gotthardt "Active modification of the vibration frequency of a polymer beam using shape-memory-alloy fibres" Proc. of $3^{\text {rd }}$ Int. Conf. on Intell. Materials, Lyon, pp. 517-522, 1996

[7] J.-E. Bidaux, W. J. Yu, R. Gotthardt, J.-A. E. Manson "Modelling of the martensitic transformation in shape memory alloy composites" J. de Physique IV, $\underline{5}$, C2-pp. 543-548, 1995

[8] J. A. Balta, M. Parlinska, V. Michaud, R. Gotthardt, J.-A. E. Månson, "Adaptive composites with embedded shape memory alloy wires" Proc. of the MRS Fall Meeting, Boston, 1999 (accepted).

[9] M. Parlinska, H. Clech, J. A. Balta, V. Michaud, J.-A. E. Månson and R. Gotthardt, "Adaptive composites with embedded shape memory alloys" Proceedings of the 4th European Mechanics of Materials Conference, Metz, June 26-29, 2000 (submitted to Journal de Physique IV). 